\title{
New approaches to Limiting
}

\author{
Philip L. Roe, Tyler B. Lung ${ }^{\dagger}$ and Jungyeoul (Brad) Maeng $\ddagger$ \\ Department of Aerospace Engineering, University of Michigan, Ann Arbor, MI 48109, USA.
}

\begin{abstract}
We introduce limiting strategies that are insensitive to the quality of the mesh. This is because they are based on the time history of a single cell.
\end{abstract}

\section{Introduction}

Some form of nonlinear limiting is of course compulsory for any numerical method that can be applied with better than first order accuracy. But the famous Godunov theorem that proclaims this says nothing about what form that nonlinearity should take. Broadly three approaches have been taken

- Artificial viscosity,

- Flux-corrected transport,

- Gradient limiting.

Of these, artificial viscosity was first employed by von Neumann and Richtmyer. It has been adopted in a sophisticated form by Jameson, but the general experience is that the adjustable parameters are not universal. We do not consider this possibility.

Flux Corrected Transport was introduced by Boris and Book. The idea is to have available two different schemes, one that is accurate and another that is believed not to introduce spurious features, but is necessarily first-order and diffusive. For efficiency it should be very similar to the accurate scheme in its structure. If we were to take one step with the cautious scheme and then add back in the missing terms everywhere we would of course obtain the accurate result. Flux Corrected Transport adds back the missing terms in full only when it is safe to do so according to some criterion. Because they are added to the flux, they take from one cell and add to another in such a way as to sharpen existing gradients.

Gradient limiting originated with the MUSCL scheme proposed by van Leer $^{7}$ In a finite volume scheme the data consists solely of cell average values, and in order to obtain better than first-order results, more than this is needed. In the original version only a gradient was added, by constructing two more or less equally plausible candidate gradients and then taking a nonlinear average, such as the harmonic mean, that is biased toward the smaller alternative. van Leer gave an appealing graphical interpretation of this, allowing a visual proof that this procedure could not create overshoots. This results in a reconstruction that is generally discontinuous across cell boundaries. Most writers, including van Leer, have taken these discontinuities literally, introducing Riemann solvers to resolve them. This is not harmful in one dimension, because it simply results in waves that travel either left or right, not differently from other waves in the solution. However, in more than one dimension, taking the discontinuous reconstruction to be genuine, introduces strong one-dimensional waves that travel perpendicular to the arbitrary cell boundary, and may have no counterpoint in the actual flow. Various authors have generalized this procedure to include higher-order reconstructions, in which the higher derivatives are also evaluated in a way that is biased toward caution.

A further classification of limiting turns on how much information is available about constraints on the solution. For example, neither density nor pressure may be negative, and speeds may not exceed the speed of light. In one-dimensional hyperbolic systems, if they are linear, the Riemann invariants may not take on new values. In pure advection problems, the value at $(\mathbf{x}, t)$ is bounded by the values along the particle path

*Professor, Fellow, AIAA

${ }^{\dagger}$ Graduate Student, Member AIAA

$\ddagger$ Graduate Student, Member AIAA 
traced backward through that point. Where bounds are available, the limiter can be designed to respect them. However, in many, perhaps most, situations no a priori bounds are available. For example, waves in many dimensions may focus and produce pressures enormously greater than those initially present, even with linear physics. ${ }^{a}$ Limiting is made much more difficult if no limits are available.

In this paper we will introduce three novel kinds of limiter. One is designed around the existence of bounds; the others are not. They do share a common feature, however, in that both derive their information from extremely compact regions of space, in one case, just a single point. We believe that such an approach is necessary if the object of designing "high-order" methods is to allow the use of coarse grids. Another strong point is that any influence of the mesh geometry is minimized.

One incidental point is that we do not regard very small overshoots, around one percent, as unacceptable. Perhaps there are examples where such a small overshoot might trigger a premature reaction, say, but generally the effect of other modeling errors, ignorance of physical constants, or simply truncation error, are likely to be at least equally blameworthy. Therefore we put more stress on maintaining a compact stencil than on exactly satisfying bounds

\section{Limiting from the Past; the Active Flux scheme.}

This scheme, like the Discontinuous Galerkin method, has a very compact stencil. It was introduced by van Leer as Scheme V in his1977 paper. ${ }^{\text {? }}$ The reconstruction within a cell depends only on local data, and is therefore independent of neighboring cells. It would be nice to have a limiter that shared these properties. We will consider the simple case of linear advection in one dimension.

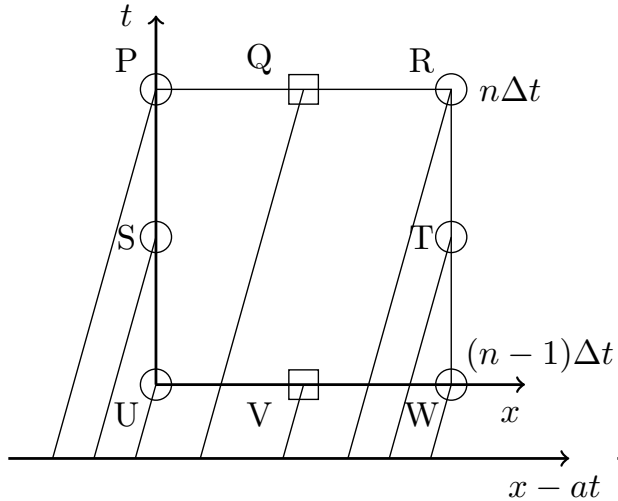

Figure 1. $\quad \nu<0.5$

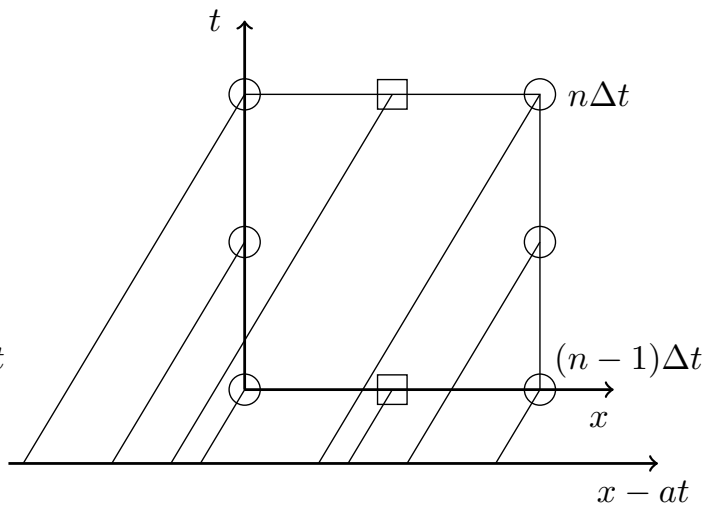

Figure 2. $\nu>0.5$

Figure 3. One dimensional characteristic coordinate

The degrees of freedom assigned to each cell in this method the cell average value, denoted in Figure(??) as $\mathrm{Q}$ at the time level $n \Delta t$ and the interface fluxes at each time level (P,R at time level $n \Delta t$ ). To advance to the time level $(n+1) \Delta t$, the unlimited scheme makes a parabolic reconstruction of this data. Then characteristics are traced backward from the new time level to determine by interpolation the new value on the right boundary (we are supposing $a>0$ ) and by integration the amount of substance that will cross that boundary. The method is third-order, explicit, fully discrete, and maximally stable. Because the method has well-matched dispersion and dissipation properties, the oscillations that it produces are small, as we will see, but in most applications do need to be removed by limiting.

The limiting strategy is to retain a memory of the previous time step, between $(n-1) \Delta t$ and $n \Delta t$. At the previous time level the cell average was at $\mathrm{V}$, and the boundary values were at $\mathrm{U}$ and $\mathrm{W}$. Also, boundary values at $\left(n+\frac{1}{2}\right) \Delta t$ were found at $\mathrm{S}$ and $\mathrm{T}$. All of these values are, in the exact solution, derived from the same function of the characteristic coordinate $\xi=x-a t$. We may make use of them, therefore, to confirm the legitimacy of our reconstruction. We refer to the figure 3 to choose the data that would give the strongest confirmation.

${ }^{a} \mathrm{~A}$ useful practical heuristic is to repeat the calculation on a differently spaced grid. If the wavelength of the oscillations scales with the mesh spacing, then they are spurious, otherwise they are real. It could be feasible to automate this test. 
The natural choices depend on whether the Courant number is less than or greater than 0.5. Such choices are shown in table ??. These choices of alternative stencils are based on their effective range in the coordinate $\xi$. We would ideally want these to occupy a range similar to that of the default stencil.

Table 1. Possible alternative stencil choices

\begin{tabular}{cc|c} 
Stencil & $\nu<0.5$ & $\nu>0.5$ \\
\hline \hline$n$, Default & PQR & PQR \\
$n-1$ & UVW & UVW \\
Alternate 1 & PSU & SUV \\
Alternate 2 & RTW & RTW \\
\hline
\end{tabular}

\section{A. Maximum Principle Satisfying (MPS) Limiter}

Our first limiting process makes a straightforward application to this data of the method proposed by Zhang and Shu, ${ }^{10,11}$ who have proposed limited high-order schemes for scalar conservation laws in one- and multidimensions, assuming that there exists some "maximum principle" that

$$
m=\max _{j} u_{j}^{n+1} \leq u_{j}^{n+1} \leq \max _{j} u_{j}^{n}=M
$$

For example, in our case, in a scalar conservation law, $M$ and $m$ are maximum and minimum values observed at the previous time level. They must not be taken from the previous cell averages, because that would correspond to the TVD criterion that limits the scheme to second-order accuracy. They are estimated instead from the reconstruction of the previous data. We replace the piecewise natural quadratic reconstruction $u(\xi)$ with a limited reconstruction $\tilde{u}(\xi)$ that satisfies known local a priori bounds.

$$
\tilde{u}(\xi)=\theta\left(u(\xi)-\bar{u}^{n}\right)+\bar{u}^{n}
$$

with the scaling coefficient

$$
\theta=\min \left(\left|\frac{M-\bar{u}^{n}}{M_{p}-\bar{u}^{n}}\right|,\left|\frac{m-\bar{u}^{n}}{m_{p}-\bar{u}^{n}}\right|, 1\right)
$$

where maxima and minima of the numerical solution and approximation polynomial are defined respectively as

$$
m=\min \left(u_{j, \text { numerical }}\right), \quad M=\max \left(u_{j, \text { numerical }}\right)
$$

and

$$
m_{p}=\min \left(u_{j}^{n}(\xi)\right), \quad M_{p}=\max \left(u_{j}^{n}(\xi)\right) .
$$

The approximation polynomial extrema are sampled at Gaussian quadrature locations.

\section{B. Results}

The Zalesak ${ }^{9}$ suite of test profiles is presented in Fig. 6 after advancing by one circuit at $\nu=0.5$. One striking feature is that the unlimited solution is already quite good, perhaps for some purposes even acceptable; this obviously makes the task of the limiter much easier. Even the notoriously difficult "semi-ellipse" profile is handled very well.

\section{Modified reconstruction (RS) limiter}

Here we take a very different approach. While retaining the interpretation of the cell data as an average value plus two boundary values, we make a reconstruction that is not a straightforward single parabola. To keep the process of interpolation straightforward, the new reconstruction is some combination of parabolas and straight lines.

We begin by observing, after some easy algebra, that the default reconstruction (the parabola that exactly matches the given cell average and boundary values) will have an internal extremum if the parameter

$$
R=\frac{u_{R}-\bar{u}}{\bar{u}-u_{L}}
$$




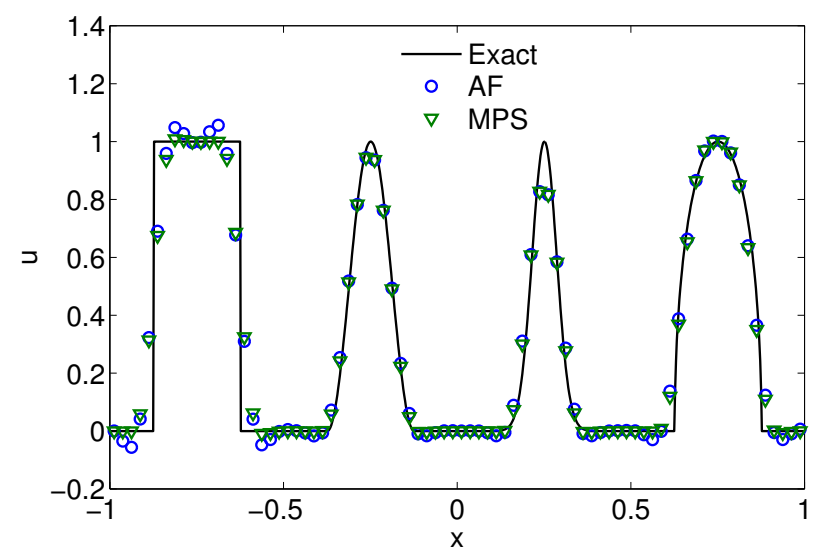

Figure 4. Zalesak test cases calculated on a mesh of 80 cells after one circuit at $\nu=0.5$.

lies outside the range ${ }^{\mathrm{b}} 1 / 2<R<2$. If the value of $R$ is in fact inside these bounds then there is no internal extremum and the default reconstruction is accepted. If it is outside, then we examine the alternative stencils to see whether the existence of such an extremum can be justified. At the moment we have only an empirical criterion for this. We calculate the second derivative from three alternative stencils, obtaining a total of four possible second derivatives. If any of these agrees with the default curvature to within a factor of two, we accept the extremum as genuine. If not, we "flatten" the reconstruction, which is modified as shown in the following figure.If $1 / 5<R<5$ the flattened reconstruction is monotone. Otherwise, it has a reduced extremum except in the case $R=-1$.
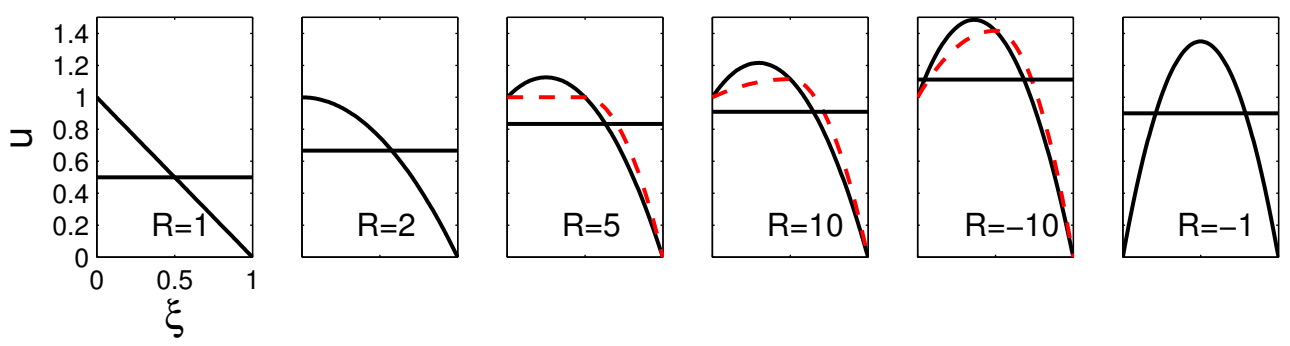

Figure 5. Modified reconstructions. For $1<R<2$ the default reconstruction is accepted.If an internal extremum with $2<R<5$, the reconstruction is a constant region plus a parabola. For $5<R<\infty$ or $0<R<1 / 2$ the reconstruction is a parabola plus a straight line tangent.

The modified reconstruction preserves both the cell average and the boundary values. Results from this method at $\nu=0.5$ are displayed in Figure ??. They are hard to distinguish from the first strategy, and we conclude from this that rigorous bounds are not required to produce acceptable behavior. To demonstrate that both methods produce results that are largely independent if Courant number, plots for $\nu=0.4,0.8$ are provided that show both methods on the same graph. Again, the methods are hard to distinguish, either one would work well in practice.

\section{Limiting in the future; the Lax Wendroff method}

One view of limiting is that every method has a default stencil, but sometimes we are not confident that the information contained in it describes a well-resolved feature and is therefore to be trusted. To check on this, we need to look outside the default stencil. and the usual way to do this is to look at neighboring data. Another way is to take a tentative step forward in time and then revaluate the time derivative. The new value will be taken from data that has been modified by neighbors of the default stencil. It does not tell us

\footnotetext{
${ }^{\mathrm{b}}$ Note that taking the reciprocal or Rjust gives the same data in reverse order. As $R$ passes through 0 (or infinity) the sequence of patterns sufferes no discontinuity.
} 


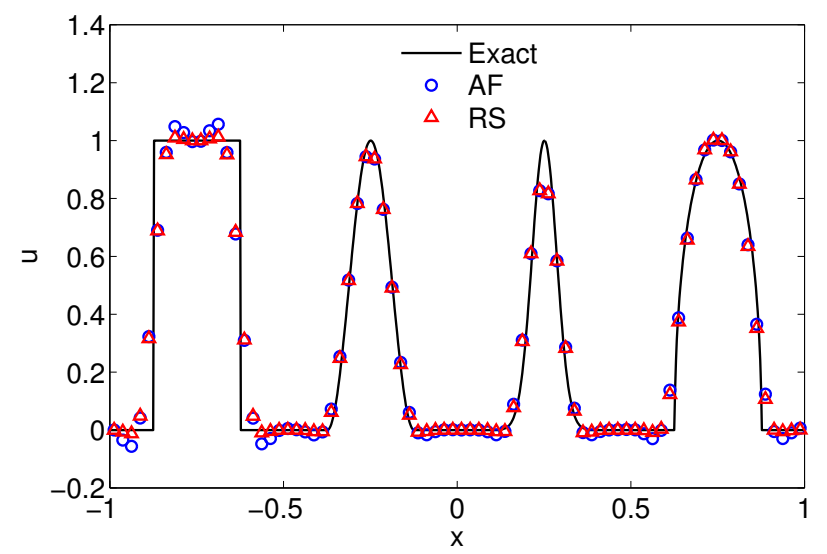

Figure 6. Zalesak test cases calculated on a mesh of 80 cells after one circuit at $\nu=0.5$.
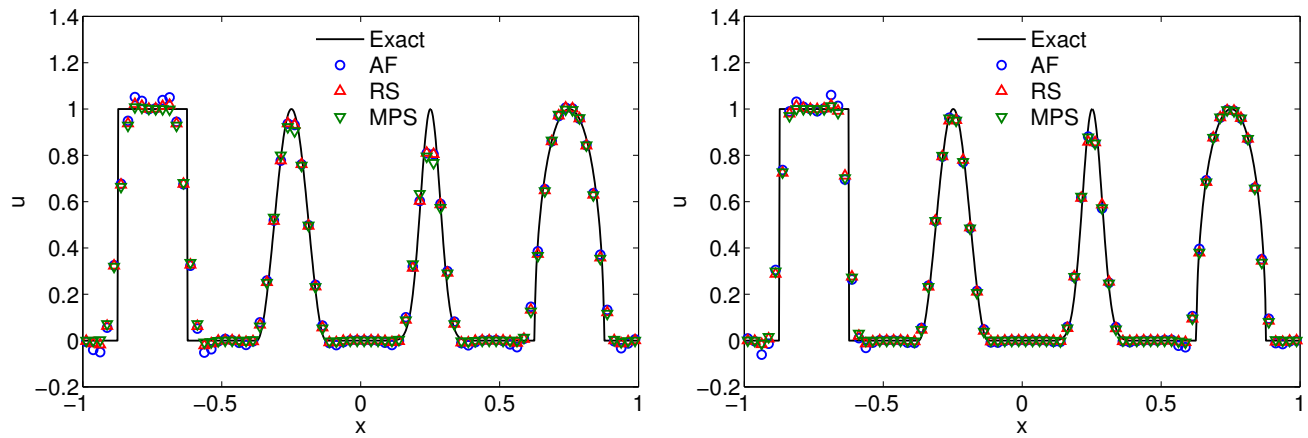

Figure 7. Zalesak test cases calculated on a mesh of 80 cells after one circuit at $\nu=0.4$ (left), and $\nu=0.8$ (right).

what the neighbors are, but it does tell us what effect they have.

\section{A. Choice of problem}

We will illustrate this approach with the linear acoustic system

$$
\begin{aligned}
\partial_{t} p+c \nabla \mathbf{v} & =0 \\
\partial_{t} \mathbf{v}+c \nabla p & =0
\end{aligned}
$$

This is perhaps the simplest problem that displays realistically the fundamentally multidimensional phenomena of wave spreading and wave focussing that prevent a priori estimates of bounds, or indeed of anything much. The only additional information that we can glean is that "energy" c $E=\frac{1}{2}\left(p^{2}+\mathbf{v} \cdot \mathbf{v}\right)$ is conserved

$$
\begin{aligned}
\partial_{t} E & =c\left(p \partial_{t} p+\mathbf{v} \cdot \partial_{t} \mathbf{v}\right) \\
& =-c(p \nabla \cdot \mathbf{v}+\mathbf{v} \cdot \nabla p) \\
& =-\nabla \cdot(p \mathbf{v})
\end{aligned}
$$

We can design numerical methods that are energy-decreasing, but unfortunately this criterion admits the regular Lax-Wendroff scheme, which certainly admits unwanted oscillations. However, different kinds of constraint can be enforced. For example, solutions to (7) preserve their initial vorticity $\partial_{t} \omega=0$, where $\omega=\partial_{x} v-\partial_{y} u$, and a discrete vorticity is preserved if the velocity updates are performed using pressure fluxes evaluated at the vertices. ${ }^{4}$

We have experimented with a simple heuristic, in which we regard successive terms in the Lax-Wendroff expansion as terms in the Taylor series, which indeed they are. If they diminish quickly, we will naively assume that the series converges and make use of it.

${ }^{\mathrm{c}}$ Some might prefer to describe this as entropy conservation. 
The series development of (??) is that

$$
\mathbf{u}(t)=\left(\mathbf{I}-c \mathbf{A} \Delta t+\frac{1}{2} c^{2} \mathbf{A}^{2}+\frac{1}{6} c^{3} \mathbf{A}^{3} \ldots\right) \mathbf{u}(0)
$$

where

$$
\mathbf{A}=\left(\begin{array}{ccc}
0 & \partial_{x} & \partial_{y} \\
\partial_{x} & 0 & 0 \\
\partial_{y} & 0 & 0
\end{array}\right), \quad \mathbf{A}^{2}=\left(\begin{array}{ccc}
\partial_{x}^{2}+\partial_{y}^{2} & 0 & 0 \\
0 & \partial_{x}^{2} & \partial_{x y}^{2} \\
0 & \partial_{x y}^{2} & \partial_{y}^{2}
\end{array}\right), \quad \mathbf{A}^{3}=\nabla^{2} \mathbf{A}
$$

A numerical method must provide some discrete form of these matrices. If we stop at second order and insist on the obvious symmetries, there are four free parameters and the numerical update is

$$
\mathbf{T}_{\Delta}=\left[\begin{array}{ccc}
\mathbf{u}^{n+1}=\mathbf{u}^{n}-\mathbf{T}_{\Delta} \mathbf{u}^{n} & \\
\frac{\nu^{2}}{2}\left(\delta_{x}^{2}\left(1+\alpha_{2} \delta_{y}^{2}\right)+\delta_{y}^{2}\left(1+\alpha_{2} \delta_{x}^{2}\right)\right) & -\nu \mu_{x} \delta_{x}\left(1+\phi_{1} \delta_{y}^{2}\right) & -\nu \mu_{y} \delta_{y}\left(1+\phi_{1} \delta_{x}^{2}\right) \\
-\nu \mu_{x} \delta_{x}\left(1+\alpha_{1} \delta_{y}^{2}\right) & \frac{\nu^{2}}{2} \delta_{x}^{2}\left(1+\phi_{2} \delta_{y}^{2}\right) & \frac{\nu^{2}}{2} \mu_{x} \mu_{y} \delta_{x} \delta_{y} \\
-\nu \mu_{y} \delta_{y}\left(1+\alpha_{1} \delta_{x}^{2}\right) & \frac{\nu^{2}}{2} \mu_{x} \mu_{y} \delta_{x} \delta_{y} & \frac{\nu^{2}}{2} \delta_{y}^{2}\left(1+\phi_{2} \delta_{x}^{2}\right)
\end{array}\right]
$$

It can be shown that vorticity will be preserved if $\phi_{2}=\alpha_{1}=1 / 4$ and that the dispersion will be isotropic to third order if, in addition $\phi_{1}=1 / 12$ It was found that $\alpha_{2}=1 / 8$ provided a good balance between isotropy and stability. The benefits gained by these choices can be appreciated from Figure 8 . The results from the original Lax-Wendroff method ${ }^{5}$ using a five-point stencil are shown for an initial-value problem consisting of a Gaussian pressure perturbation applied to a stationary flow. The exact solution should be axisymmetric but it is not easy to achieve this on a square grid. Also shown are results using the optimized parameter set just described. Although the scatter is much reduced, other faults remain, including overshoots and spurious features, which it is the task of a limiter to deal with. The problem that we want to address is how to achieve that without giving up the isotropy that has been achieved.
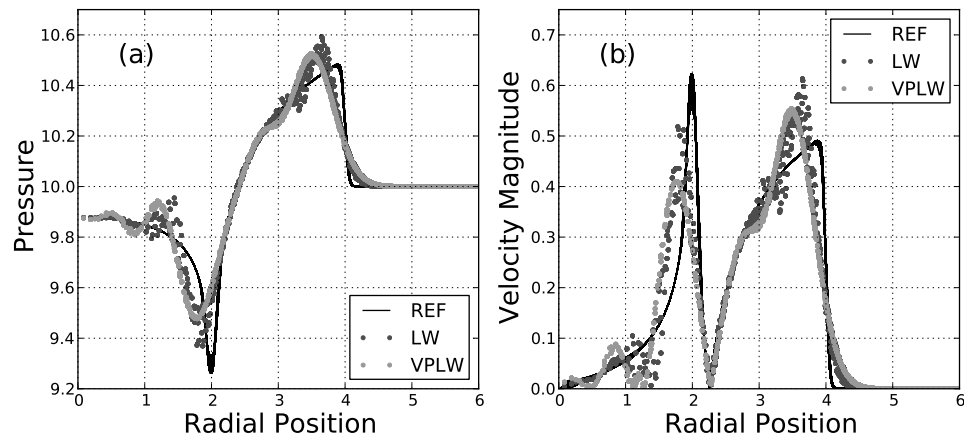

Figure 8. By selecting the free parameters in the Lax-Wendroff method, the anisotropic scatter of the original version can be greatly reduced, although isotropic oscillations remain.

Taking these choices to define the accurate scheme, we can construct low-order schemes by increasing the coefficients of the second-order terms. Recall that the family of $q$-schemes in one dimension for linear advection is given by

$$
u^{n+1}=\left(1-\nu \mu \delta+\frac{1}{2} q(\nu) \delta^{2}\right) u^{n}
$$

where $q=\nu^{2}$ is the smallest amount of dissipation that will results a stable scheme, and simultaneously the only value that gives second-order accuracy. Recognized low-order schemes include $q(n u)=|\nu|$ (First-order upwind), $q(\nu)=\left(1+2 \nu^{2}\right) / 3$ (Low phase error) and $q(\nu)=1.0$ (Lax-Friedrichs). By analogy, we construct a low-order two-dimensional scheme by replacing the factor $\nu^{2}$ by $q(\nu)$ wherever it appears in (??)

$$
\mathbf{T}_{\Delta}^{C}=\left[\begin{array}{ccc}
\frac{q(\nu)}{2}\left(\delta_{x}^{2}\left(1+\frac{1}{8} \delta_{y}^{2}\right)+\delta_{y}^{2}\left(1+\frac{1}{8} \delta_{x}^{2}\right)\right) & -\nu\left(1+\frac{1}{12} \delta_{y}^{2}\right) \mu_{x} \mu_{y}^{2} \delta_{x} & -\nu\left(1+\frac{1}{12} \delta_{x}^{2}\right) \mu_{y} \delta_{y} \\
-\nu \mu_{x} \mu_{y}^{2} \delta_{x} & \frac{q(\nu)}{2} \mu_{y}^{2} \delta_{x}^{2} & \frac{q(\nu)}{2} \mu_{x} \mu_{y} \delta_{x} \delta_{y} \\
-\nu \mu_{x}^{2} \mu_{y} \delta_{y} & \frac{q(\nu)}{2} \mu_{x} \mu_{y} \delta_{x} \delta_{y} & \frac{q(\nu)}{2} \mu_{x}^{2} \delta_{y}^{2}
\end{array}\right]
$$


We are not claiming that this is in any way an optimal choice of cautious scheme, but it does have in common with the accurate the property of preserving vorticity and having isotropic dispersion error. It would be a shame to lose these properties in the process of limiting. We can ensure that they are not lost by making the limited scheme a linear combination ${ }^{\mathrm{d}}$ of schemes that all have the desired properties. In this instance the family of schemes is described by the single parameter $q(\nu)$ but a broader basis is possible. Our limited scheme will begin by taking one cautious time step, employing a cautious choice $q^{C}(\nu)$.

$$
\mathbf{u}^{*}=\mathbf{u}^{n}-\mathbf{T}_{\Delta}^{C} \mathbf{u}^{n}
$$

and continue by adding back the remaining second-order terms, which we will call the anti-diffusive terms.

$$
\mathbf{u}^{n+1}=\mathbf{u}^{*}-\left(\mathbf{T}_{\Delta}-\mathbf{T}_{\Delta}^{C}\right)\left(\kappa \mathbf{u}^{*}+(1-\kappa) \mathbf{u}^{n}\right)
$$

where $\kappa$ is to be determined.

\section{B. What gets limited}

We can write the antidiffusive part of the update as

$$
\frac{q(\nu)-\nu^{2}}{2}\left[\delta_{x} \mathbf{F}^{A D}+\delta_{y} \mathbf{G}^{A D}\right]
$$

where the $\mathbf{F}^{A D}, \mathbf{G}^{A D}$ are antidiffusive fluxes. They are

$$
\mathbf{F}^{A D}=\left(\begin{array}{c}
\left(1+\frac{1}{8} \delta_{y}^{2}\right) \delta_{x} p \\
\mu_{y}\left(\mu_{y} \delta_{x} u+\mu_{x} \delta_{y} v\right) \\
0
\end{array}\right), \quad \mathbf{G}^{A D}=\left(\begin{array}{c}
\left(1+\frac{1}{8} \delta_{x}^{2}\right) \delta_{y} p \\
0 \\
\mu_{x}\left(\mu_{y} \delta_{x} u+\mu_{x} \delta_{y} v\right)
\end{array}\right)
$$

These are proportional to the pressure gradient and to the velocity divergence, which are of course, the only quantities to bring about changes in the flow. We call them the driver quantities, and refer to them as $\beta_{u}, \beta_{v}, \beta_{p}$ respectively, thus

$$
\mathbf{F}^{A D}=\left(\begin{array}{c}
\left(1+\frac{1}{8} \delta_{y}^{2}\right) \beta_{u} \\
\mu_{y} \beta_{p} \\
0
\end{array}\right), \quad \mathbf{G}^{A D}=\left(\begin{array}{c}
\left(1+\frac{1}{8} \delta_{x}^{2}\right) \beta_{v} \\
0 \\
\mu_{x} \beta_{p}
\end{array}\right)
$$

Looking back at (13) we can see that the drivers could be evaluated either at the old time level or after the provisional step. This a typical situation for limiting; there is a choice of alternative estimates and we can choose the one that reduces the potential for nonphysical solutions. First, however, we will establish the most accurate choice.

\section{A third-order option}

There is a choice of $\kappa$ that will give third-order accuracy. This was established by a dispersion analysis but can be demonstrated in a non-rigorous way by analogy with the linear advection equation, for which the proposed predictor-corrector method would give

$$
\begin{aligned}
u^{n+1} & =\left(1-\nu \mu \delta+\frac{q^{C}}{2} \delta^{2}+\frac{\nu^{2}-q^{C}}{2} \delta^{2}\left(\kappa\left(1-\nu \mu \delta+\frac{q^{c}}{2} \delta^{2}\right)+(1-\kappa)\right)\right) u^{n} \\
& =\left(1-\nu \mu \delta+\frac{\nu^{2}}{2} \delta^{2}-\frac{\kappa \nu\left(\nu^{2}-q^{C}\right)}{2} \mu \delta^{3}\right) u^{n}
\end{aligned}
$$

Or, representing the operators by their Fourier symbols, $\delta=2 i \sin \frac{1}{2} \theta, \mu=\cos \frac{1}{2} \theta$ and expanding in powers of $\theta$,

$$
g=\left(1-i \nu \theta-\frac{1}{2} \nu^{2} \theta^{2}+\left(\frac{1}{6} i \nu+\frac{1}{2} i \kappa \nu\left(\nu^{2}-q\right)\right) \theta^{3}\right.
$$

\footnotetext{
${ }^{\mathrm{d}}$ Although the scheme is a linear combination, the weighting will be determined by a linear process.
} 
For this to match with the true expansion (9), we must have

$$
\kappa=\frac{1-\nu^{2}}{3\left(q^{C}-\nu^{2}\right)}
$$

For particular choices of the cautious scheme, we have, for First-order upwinding, $\kappa=(1+\nu) /(3 \nu)$, for the Low Dispersion Error method, $\kappa=1$, and for Lax-Friedrichs, $\kappa=1 / 3$.

\section{Nonlinear limiting}

Each driver quantity $\beta$ will be written as

$$
\beta^{\lim }=\beta^{n}-\kappa\left(\beta^{*}-\beta^{n}\right)
$$

and in smooth regions the choice of $\kappa$ given above will provide third-order accuracy. By itself, this brings about a substantial improvement. The abiding problem with second-order methods is their large phase error combined with a lack of damping at high frequencies. The third-order method improves the phase error, so that the lightly damped waves now propagate at the proper speeds. Figure 9 shows the great improvement
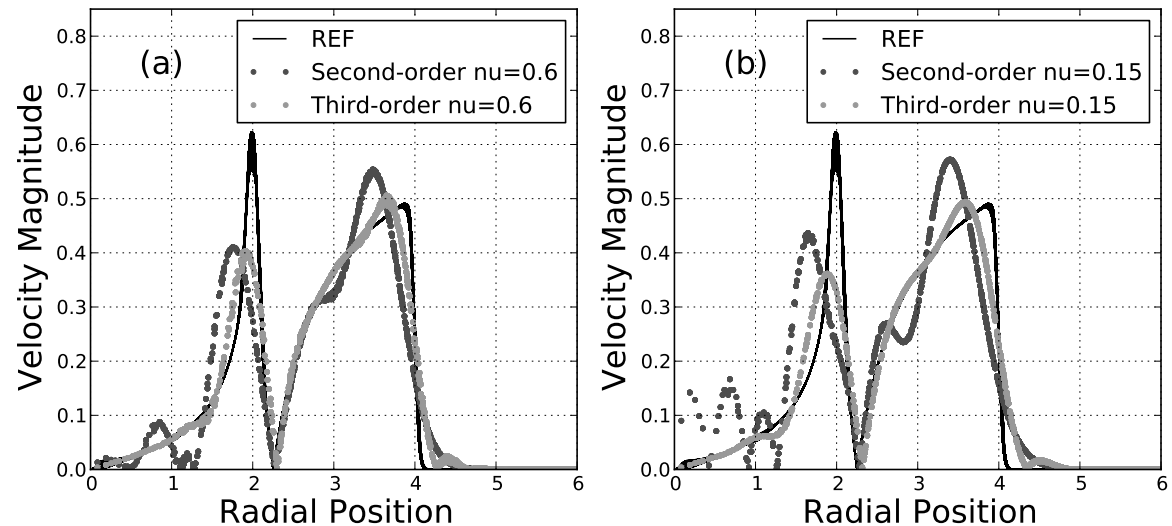

Figure 9. Comparison of Second- and Third-Order schemes. The reference solution isa MUSCL-Hancock method on a $600 \times 600$ mesh

produced. It might be said that the solution is now better prepared for the limiter.

We now introduce a nonlinear dependence by writing

$$
\beta^{\lim }=F_{0}(\phi, n u)+F_{1}(\kappa, \phi, n u)\left(\beta^{*}-\beta^{n}\right)
$$

A novelty here is the quantity $\phi$ intended as an indicator of local difficulty. The idea is that perhaps no antidiffusion is justified in badly-behaved regions, but that in regions of less difficulty the functions $F_{0}, F_{1}$ should respectively approach 1 and $\kappa$ to give the third-order result.

The argument to define $\phi$ proceeds by expanding the pressure that is used as a vertex flux as

$$
p(t) \simeq p^{n}+t \partial_{t} p+\frac{t^{2}}{2} \partial_{t t}^{2} p
$$

or, expressed in terms of the driver $\beta_{p}$,

$$
p(t) \simeq p^{n}-\frac{t a_{0}}{h} \beta_{p}^{n}-\frac{t^{2} a_{0}}{h}\left(\partial_{t} \beta_{p}\right)^{n}
$$

It will be taken as a danger signal if the third term approaches or exceeds the second in absolute value, i.e.

$$
\phi_{p}=\left|\frac{\Delta t^{2} a_{0} \partial_{t} \beta_{p}}{a_{0} \Delta t \beta_{p}^{n}}\right| \simeq\left|\frac{\beta_{p}^{*}-\beta_{p}^{n}}{2 \beta_{p}^{n}}\right|
$$


is in some sense large.

Remembering that we are in a situation where there may be no rigorous argument available, we take

$$
F_{0}=\max [0,1-f(\nu) \phi]
$$

If the situation is perceived to be easy, $\phi$ will be small and $F_{0}$ will be close to unity. As the situation becomes harder, $F_{0}$ is reduced, eventually to zero. Quite good results were obtained with this choice of $F_{0}$ and with $F_{1}=\kappa$ but useful improvements were obtained by taking

$$
F_{1}=\kappa \min \left(\frac{f(\nu)}{\phi}, 1\right)
$$

shown in Figure 10 so that large values of $\phi$ would remove some of the antidiffusive effects in this term. The functions $f(\nu)$ were taken to be $f(\nu)=3(1-\nu) / 2$ if the cautious scheme was first-order upwinding, and $3 \nu / 2$ for the LPE scheme. The solution obtained with these choices is shown in Figure ??.
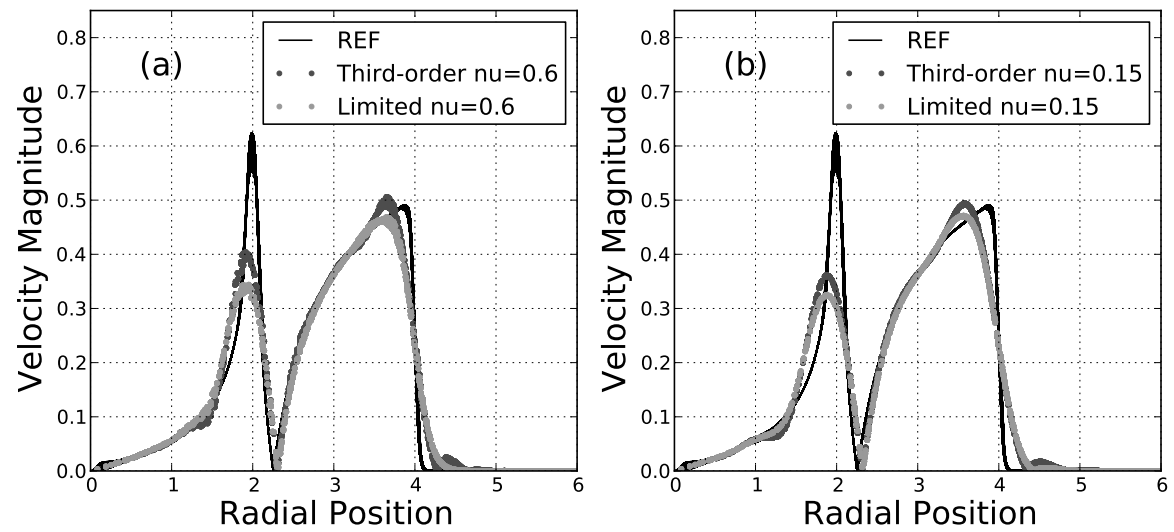

Figure 10. The limited solution is accurately isotropic and free of spurious features

There is obviously a lot of empiricism in these choices. Some of the experimentation that led to them is described in the thesis of the second author. ${ }^{3}$ We wish to state however, that no choice was very critical. Others could have been made, provided that they produced similar trends. The striking result is that we were able to generate results that were considerably better than those produced by standard one-dimensional arguments. Figure ?? shows results for the smooth exact solution

$$
\begin{aligned}
& p(x, y, t)=\frac{1}{c} \cos 2 \pi c t[\sin 2 \pi x+\sin 2 \pi y] \\
& u(x, y, t)=\frac{1}{c} \sin 2 \pi c t \cos 2 \pi x \\
& v(x, y, t)=\frac{1}{c} \sin 2 \pi c t \cos 2 \pi y
\end{aligned}
$$

This was solved with all limiters in place, and compared with the standard MUSCL-Hancock procedure using either the Minmod or Superbee limiters. Finally we show results for a problem in which four shockwave are located in a box in such a way that the exact solution has them restored to their original locations after each reflection (see Figure ??

\section{Conclusions}

We have drawn attention to some novel methods of providing nonlinear limiting. Although these methods are not yet fully mature, and can probably be improved, they have features that are desirable and worth preserving. Chiefly, they make use of information that is spatially very compact, being based on either a single cell or a single vertex. This avoids undue dependence on mesh smoothness and helps to preserve properties that have been built into the base method. 


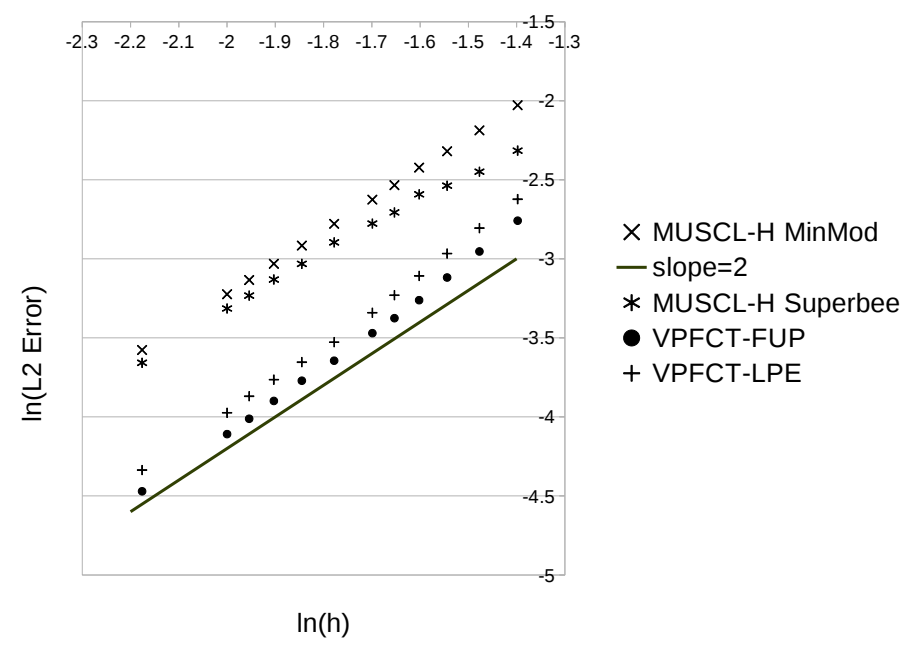

Figure 11. The limited solution converges to second-order on a smooth problem and is considerably more accurate than standard dimensional upwinding,

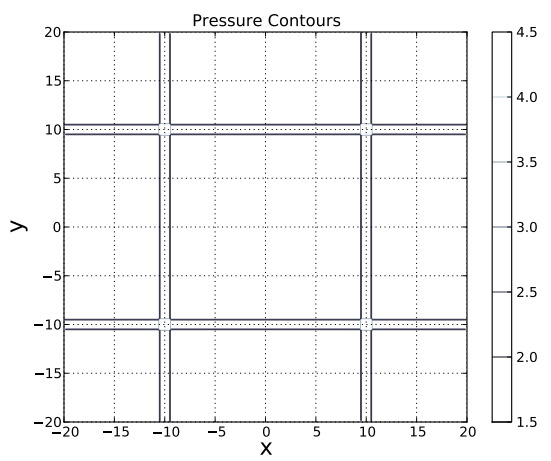

Figure 12. Four linear regions of high pressure are located so that each will give rise to a pair of waves travelling in opposite directions. One of these waves will reflect off the wall and return to its initial location. The other will, in the same time, arrive at the initial location of its "partner". The result is to restore the initial configuration. 

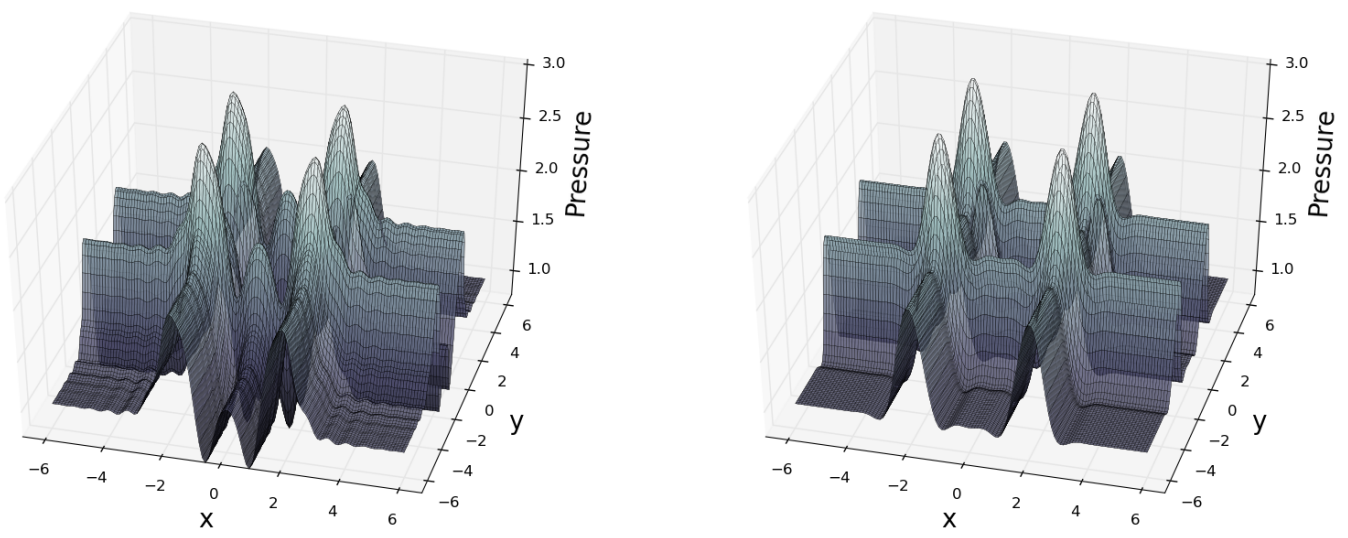

Figure 13. Two solutions to problem of plane shock reflection against a background flow with vorticity. At left is a second-order scheme without limiting. At right the limiting has produced a crisper solution without overshoots.

\section{References}

${ }^{1}$ J. P. Boris, D.L. Book, Flux-corrected transport, I, SHASTA, a fluid transport algorithm that works, J. Comput. Phys, 11(1), pp 38-69, 1973.

${ }^{2}$ J. P. Boris, D.L. Book, Flux-corrected transport, III, minimal-error FCT algorithms, J. Comput. Phys, 20(4), pp 397-431, 1976.

${ }^{3}$ Lung, T. B., Toward a simple, accurate, Lagrangian hydrocode, thesis, Department of Aerospace Engineering, University of Michigan, 2015.

${ }^{4}$ K. W. Morton, P. L. Roe, Vorticity-preserving schemes for the system wave equation, SIAM J. Sci. Comp., 23(1), 2001.

${ }^{5}$ P. D. Lax, B. Wendroff, Systems of conservation laws, Comm. Pure and Applied Math., 7, 1960.

${ }^{6} \mathrm{~B}$. van Leer, Towards the ultimate conservative differencing scheme, II. Monotonicity and conservation combined in a second-order scheme, J. Comput Phys., 14(4), 1974.

${ }^{7}$ B. van Leer, Towards the ultimate conservative differencing scheme, IV. A newapproach to numerical convection, $J$. Comput Phys., 23(3), 1974.

${ }^{8}$ J. von Neumann, R. D. Richtmyer, A method for the numerical calculation of hydrodynamic shocks, J. Apl. Phys, 21(3), 1950.

${ }^{9}$ S.T. Zalesak, A preliminary comparison of modern shock-capturing schemes: linear advection. In: R. Vichnevetsky and R. Stepleman (eds.) Advances in Computer Methods for PDEs. Publ. IMACS, 15-22, 1987.

${ }^{10} \mathrm{X}$. Zhang, C-W. Shu, On maximum-principle-satisfying high order schemes for scalar conservation laws, J. Comput. Phys., 229(9), 2010.

${ }^{11} \mathrm{X}$. Zhang, C-W. Shu, Maximum-principle-satisfying and positivity-preserving high-order schemes for conservation laws: survey and new developments. Proc. R. Soc. A 2011. 